\title{
Recuperação de modelos médicos por conteúdo usando Extrator Local de Área e Distância
}

\author{
Pedro H. M. Delmondes ${ }^{1}$ \\ Leila C. C. Bergamasco ${ }^{2}$ \\ Fátima L. S. Nunes ${ }^{1}$
}

Data submissão: 24.02 .2015
Data aceitação: 05.06 .2015

\begin{abstract}
Resumo: Sistemas de Recuperação de Imagens por Conteúdo têm sido desenvolvidos para contribuir com o diagnóstico em Medicina. Apesar de largamente explorados para imagens bidimensionais, seu uso com modelos tridimensionais ainda é incipiente. Este artigo tem o objetivo de introduzir o novo extrator de caraterísticas ELAD (Extrator Local de Área e Distância) visando à recuperação por conteúdo, com base na área e no posicionamento das faces da malha de modelos tridimensionais. $\mathrm{O}$ extrator foi aplicado em modelos médicos que representam o ventrículo esquerdo do coração para auxiliar o diagnóstico de Insuficiência Cardíaca Congestiva (ICC). Os resultados obtidos são comparados com resultados de um extrator baseado na Transformada de Hough 3D (ETH3D) desenvolvido em um trabalho anterior. O novo extrator atingiu mais de $85 \%$ de precisão, em todos os valores de revocação, para modelos diagnosticados com ICC e valores acima de $70 \%$, em todos os valores de revocação, para modelos saudáveis.
\end{abstract}

\begin{abstract}
Content-Based Image Retrieval systems have been developed to aid diagnosis in Medicine. These systems are well explored for two-dimensional medical images, but there are few studies with three-dimensional medical models. In this article we present the LADD (Local Area-Distance Descriptor), a descriptor for contentbased retrieval, based on the area and position of the faces of three-dimensional models. This descriptor was applied to medical models, specifically in models of the heart's left ventricle, aiming at aiding the diagnosis of Congestive Heart Failure (CHF). The results are compared to the results from another descriptor based on the 3D Hough Transform (ETH3D). The new descriptor presented in this article reached over $85 \%$ of accuracy, for all recall values, for the models with ICC. For healthy models, the accuracy reached was of more than $70 \%$, for all recall values.
\end{abstract}

\footnotetext{
${ }^{1}$ Laboratório de Aplicações de Informática em Saúde (LApIS), Escola de Artes Ciências e Humanidades, Universidade de São Paulo

pedro.delmondes@usp.br, fatima.nunes@usp.br

${ }^{2}$ Escola Politécnica, Universidade de São Paulo

leila.cristina@usp.br
} 


\section{Introdução}

Os exames que obtêm imagens das estruturas internas do corpo humano, como, por exemplo, Radiografia e a Ressonância Magnética, geram um grande volume de imagens que são armazenadas e requerem técnicas eficazes de pesquisa para sua recuperação. A Recuperação de Imagens Baseada em Conteúdo (CBIR - Content-Based Image Retrieval) tem se mostrado uma abordagem válida para esta questão, consistindo em buscar em um banco de dados as imagens mais similares a uma imagem fornecida como argumento de busca. Nesse contexto, podem ser construídos Sistemas de Auxílio ao Diagnóstico (CAD - ComputerAided Diagnosis) fornecendo uma segunda opinião ao profissional da saúde a respeito da existência de uma determinada doença [13], por meio da disponibilização de casos similares.

Os conceitos aplicados em CBIR podem ser estendidos para o contexto tridimensional (3D), ainda pouco explorado na literatura. Também conhecida como CBIR 3D ou Model Retrieval, esta área é promissora, visto que modelos 3D podem compilar grande quantidade de informações provenientes de imagens bidimensionais e, consequentemente, podem contribuir para diminuir a quantidade de processamento necessário na recuperação por conteúdo, quando comparada ao processamento demandado para obter dados de todas as imagens bidimensionais que o compõem.

Uma das etapas mais importantes de um sistema CBIR 3D, similarmente a CBIR tradicional (que usa imagens bidimensionais) é a extração e a comparação de características, na qual são definidos e quantificados critérios que serão utilizados para medir a similaridade entre imagens. Apesar de existirem atualmente diferentes técnicas para extração de características de modelos tridimensionais, poucas foram aplicados na recuperação de modelos médicos $[10,15]$.

O objetivo desse trabalho é apresentar o Extrator Local de Área e Distância (ELAD) que considera a área e o posicionamento das faces de uma superfície em relação ao centro do objeto 3D no processo de comparação de similaridade. O extrator é aplicado na recuperação de modelos médicos do ventrículo esquerdo do coração, reconstruídos a partir de exames de Ressonância Magnética, com o objetivo de auxiliar o diagnóstico da doença de Insuficiência Cardíaca Congestiva (ICC). Também é apresentada uma comparação de desempenho do ELAD com o extrator ETH3D desenvolvido por Bergamasco em [2].

Esse artigo está dividido da seguinte forma: a Seção 2 explica os conceitos envolvidos na recuperação por conteúdo; na Seção 3 são mencionados trabalhos correlatos a este; a Seção 4 refere-se à Metodologia empregada, descrevendo como foram construídos os objetos síntéticos e obtidos os objetos 3D médicos do ventrículo esquerdo, além de apresentar os extratores utilizados na pesquisa e a forma de avaliação empregada; na Seção 5 os resultados obtidos são discutidos e na Seção 6 são apresentadas as considerações finais do trabalho. 


\section{Recuperação de imagens com base em conteúdo}

O funcionamento de um sistema CBIR é baseado em cinco etapas segundo Castañon [7]: pré-processamento da imagem, extração de características, definição da similaridade, visualização dos resultados e, opcionalmente, realimentação por relevância.

A etapa de pré-processamento consiste em executar procedimentos que preparem a imagem para a extração de características [24]. Nesta etapa as técnicas se dividem em níveis baixo, médio e alto. As técnicas de baixo nível atuam principalmente na correção de orientação da imagem, suavização de ruídos e realce de estruturas de interesse. No processamento de nível médio é realizada a segmentação, quando são identificadas estruturas de interesse, como bordas ou regiões da imagem. O processamento de alto nível se encarrega de agrupar os dados obtidos do processamento anterior a fim de relacioná-los a uma base de conhecimento e, assim, imprimir um significado aos dados obtidos.

Para comparar um modelo com outros, é necessário extrair características intrínsecas de cada um deles, comumente relacionadas a cor, textura ou forma. O processo de extração emprega algoritmos que quantificam os aspectos extraídos e os armazenam em vetores de características. É desejável que essa estrutura seja indexada e armazenada em um banco de dados, proporcionando melhoria no desempenho do sistema [5].

A partir da obtenção dos vetores de cada modelo, aplicam-se técnicas para comparar as características dos modelos [21]. Dentre essas técnicas destacam-se aquelas provenientes de Inteligência Artificial ou o uso de funções de distância, como a Euclidiana e a Manhattan [6, 11]. A Figura 1 ilustra o processo de Recuperação de Imagens Baseada em Conteúdo.

No processo do CBIR 3D essas mesmas etapas são aplicadas com algumas adaptações, como o uso de técnicas de pré-processamento específicas para modelos tridimensionais e extratores que identificam características exclusivas de modelos tridimensionais, como forma e volume.

\section{Trabalhos correlatos}

O desenvolvimento de extratores para modelos médicos tridimensionais ainda é escasso na literatura e não foram encontrados trabalhos que se dediquem ao auxílio de diagnóstico da Insuficiência Cardíaca Congestiva - estudo de caso empregado no presente trabalho.

No trabalho de Depeursinge et al. [12], é constatado que muitos artigos sobre recuperação de imagens médicas foram escritos nos últimos anos, porém em sua maioria os trabalhos analisam o modelo tridimensional por meio de slices ou projeções do modelo. A predominância desse tipo de trabalho é observável na revisão aqui apresentada. 


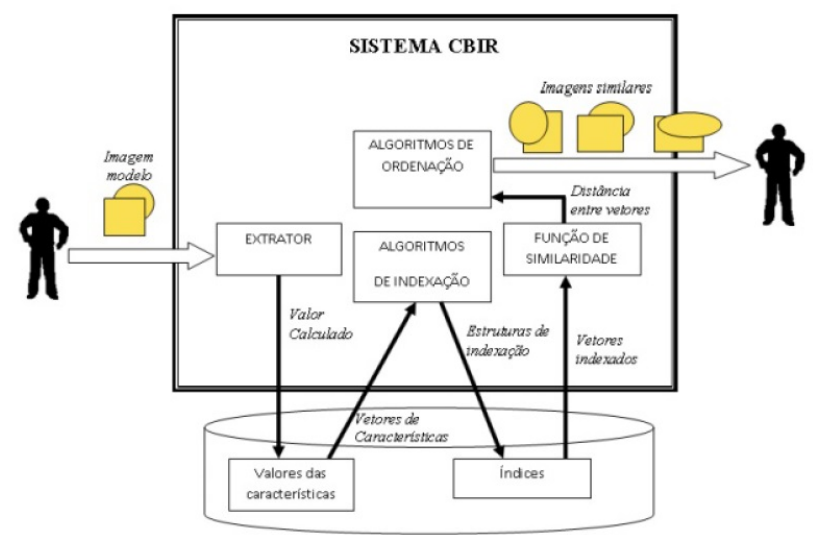

Figura 1. Processo de Recuperação por Conteúdo [23].

A partir das fatias obtidas de um volume 3D do miocárdio, Glatard et al. [14] identificaram em qual fase do ciclo cardíaco (sístole ou diástole) uma imagem de consulta estava enquadrada para tentar encontrar fatias similares à ela. Para isso, foram usados filtros de Gabor tanto para indexar a imagens quanto para para segmentá-las. Aman, Yao and Summers [1] usaram o extrator SIFT e Bag of Words para recuperar Tomografias Computadorizadas de Colonografia. Outro exemplo de trabalho que extrai características de imagens médicas 2D, foi encontrado em [22], Murala et al. propõem um método que extrai a relação da escala de cinza entre um pixel central com seus vizinhos. Essas relações são representadas em gráficos com padrões de pico e vale, possibilitando a comparação e a recuperação das imagens.

Em Wu et al. [26] foram combinados diferentes descritores de volume (VOI - Volume of Interest) para analisar modelos do cérebro provenientes do exame de Tomografia por Emissão de Prótons (PET - Positron Emission Tomography): Volume 3D, Localização do Volume 3D, Distribuição da Superfície e dados dos pacientes. Foi usada a Distância Euclidiana para comparar a similaridade entre os modelos. Também na área de PET-CT, Kumar et al. [19] fez busca de similaridade usando grafos considerando características de tumores cancerígenos como tamanho, distância, volume e localização espacial das lesões.

No trabalho de Bergamasco et al. [3] foi aplicado o Extrator de Histograma de Distâncias [20] em modelos cardíacos. Em outro trabalho de Bergamasco et al. [4] foi proposta uma modificação no Extrator de Histograma de Distâncias, na qual a técnica é aplicada separadamente em cada octante dos modelos 3D.

A Transformada de Hough, proposta em 1962 por Paul Hough, objetiva aperfeiçoar a 
detecção de retas e curvas em imagens, entretanto foi identificado no trabalho de Bergamasco [2], que a extensão dessa técnica, a Transformada de Hough 3D, proposta por Khoshelham [18] discretiza as informações de localização e grau das curvas dos objetos 3D de uma forma que pode ser utilizada na representação de modelos $3 \mathrm{D}$ por meio de um vetor de características. No trabalho citado, essa técnica adaptada (chamada de Extrator da Transformada de Hough 3D - ETH3D) foi usada no mesmo problema de recuperação de modelos cardíacos com e sem a presença de ICC. Por ser até então, o extrator que havia obtido o melhor desempenho para o conjunto de modelos, o mesmo foi usado para comparação com a atual abordagem.

Nos trabalhos analisados na literatura foram observadas duas limitações comum a todos os extratores: (1) foram aplicados em cenários muito específicos limitando o seu escopo e (2) carência de aplicação de métodos de avaliação como a métrica de Precisão vs Revocação, dificultando uma análise mais aprofundada da eficiência do extrator. Adicionalmente, os modelos citados na literatura não são disponibilizados a fim de que seja possível constituir um benchmark comum para testes. Dessa forma, o presente trabalho realiza a comparação do ELAD com o ETH3D, visto que este obteve um bom desempenho nesse cenário da ICC e foi avaliado com uma métrica conhecida em sistemas CBIR, permitindo uma comparação objetiva entre as abordagens.

\section{Metodologia}

Após a revisão bibliográfica, foi proposto e desenvolvido o ELAD que é voltado para a extração de características relativas à forma dos modelos médicos 3D (Seção 4.1). Adicionalmente, foi desenvolvido um novo método para o cálculo de similaridade entre dois modelos 3D (Seção 4.2) e, por fim, o ELAD foi validado com dois conjuntos de modelos 3D: o primeiro constituído por modelos genéricos construídos sinteticamente e o segundo de modelos reconstruídos a partir de imagens médicas reais (Seção 4.3) e utilizando matrizes de confusão e a métrica de Precisão vs. Revocação para realizar a avaliação do sistema CBIR 3D utilizando o ELAD (Seção 4.4).

\subsection{Extrator de Local Área e Distância (ELAD)}

Um modelo tridimensional convexo é descrito pela área de suas faces e orientação espacial de seu vetor normal. Ao representar essas informações em uma esfera, transformando as coordenadas do vetor normal de cartesianas para esféricas, obtemos uma Extended Gaussian Image (EGI) [16]. Porém, a maioria dos modelos 3D não são convexos. Dessa forma, para descrever esses modelos, são necessárias também informações acerca da distância de suas faces em relação ao centróide do objeto. Ao adicionar essa característica a uma EGI é 
obtido então um Global Geometric Feature Map (GGFM) [25].

Esse extrator é baseado na construção de um GGFM. Considera como características do modelo 3D a área de cada face e a distância de cada face ao centróide do modelo. A área de cada face é obtida calculando-se o comprimento das arestas que a formam. Igualmente é obtida a distância do centro da face até o centróide do modelo. Por fim são calculadas as coordenadas e o comprimento do vetor normal de cada face, que posteriormente são convertidas para coordenadas esféricas. A Figura 2 ilustra as características observadas em uma face de um modelo, na qual $D$ é a distância do centro da face ao centróide do modelo, $\theta$ e $\phi$ indicam as coordenadas esféricas do vetor normal da face, e a parte em verde indica a área da face formada pelos vértices $\mathrm{A}, \mathrm{B}$ e $\mathrm{C}$.

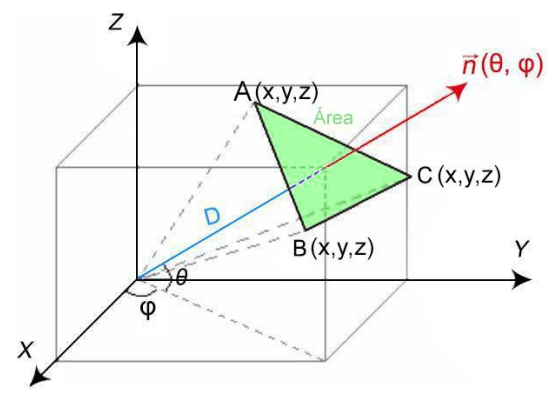

Figura 2. Exemplo das características observadas em cada face do modelo

Para a construção do GGFM define-se cada face do objeto por meio de dois vetores de características, conforme a Equação 1 , em que $F_{k}$ é a $k$-ésima face do modelo 3D; $A_{k}$ é a área da face $k ; D_{k}$ é a distância da face $k$ ao centróide do objeto; $\theta_{k}$ e $\phi_{k}$ são as coordenadas esféricas do vetor normal da face $k$; $\theta$ representa o ângulo da inclinação do vetor normal da face $k$ em relação ao plano $x y$, definido conforme a Equação 2 e $\phi$ representa o ângulo da inclinação em sentido anti-horário da projeção do vetor normal da face $k$ no plano $x y \mathrm{em}$ direção ao plano zy, definido conforme a Equação 3.

$$
F_{k}=\left[A_{k} ; \theta_{k} ; \phi_{k}\right],\left[D_{k} ; \theta_{k} ; \phi_{k}\right]
$$

O vetor normal da face $k$ é definido pelas coordenadas cartesianas $x_{k}, y_{k}$ e $z_{k}$, obtidas segundo as Equações 4, 5 e 6, respectivamente. Seu comprimento $\left(r_{k}\right)$ é calculado a partir da Distância Euclidiana entre as coordenadas do vetor normal e as coordenadas do centróide do objeto (Equação 7), na qual $x_{p}, y_{p} \mathrm{e} z_{p}$ indicam as coordenadas cartesianas do centróide do objeto. 


$$
\begin{gathered}
\theta=\arccos \left(\frac{z_{k}}{r_{k}}\right) \\
\phi=\arctan \left(\frac{y_{k}}{x_{k}}\right) \\
x_{k}=\left(y_{2}-y_{1}\right) *\left(z_{3}-z_{1}\right)-\left(z_{2}-z_{1}\right) *\left(y_{3}-y_{1}\right) \\
y_{k}=\left(z_{2}-z_{1}\right) *\left(x_{3}-x_{1}\right)-\left(x_{2}-x_{1}\right) *\left(z_{3}-z_{1}\right) \\
z_{k}=\left(x_{2}-x_{1}\right) *\left(y_{3}-y_{1}\right)-\left(y_{2}-y_{1}\right) *\left(x_{3}-x_{1}\right) \\
r_{k}=\sqrt{\left(x_{k}-x_{p}\right)^{2}+\left(y_{k}-y_{p}\right)^{2}+\left(z_{k}-z_{p}\right)^{2}}
\end{gathered}
$$

A área de cada face é calculada segundo a Equação 8, onde $\cos \alpha$ é definido pela Equação 9. $\mathrm{AB}, \mathrm{BC}$ e $\mathrm{AC}$ são os comprimentos dos segmentos de reta que formam uma face triangular, definidos, respectivamente, pelas Equações 10, 11 e 12 nas quais $x_{n}, y_{n}$ e $z_{n}$ indicam as coordenadas cartesianas do vértice $n$ da face.

$$
\begin{gathered}
A_{k}=\frac{A B * B C *\left(\sqrt{\left(1-\cos ^{2} \alpha\right.}\right)}{2} \\
\cos \alpha=\frac{A B^{2}+B C^{2}-A C^{2}}{2 * A B * B C} \\
A B=\sqrt{\left(x_{b}-x_{a}\right)^{2}+\left(y_{b}-y_{a}\right)^{2}+\left(z_{b}-z_{a}\right)^{2}} \\
B C=\sqrt{\left(x_{c}-x_{b}\right)^{2}+\left(y_{c}-y_{b}\right)^{2}+\left(z_{c}-z_{b}\right)^{2}} \\
A C=\sqrt{\left(x_{c}-x_{a}\right)^{2}+\left(y_{c}-y_{a}\right)^{2}+\left(z_{c}-z_{a}\right)^{2}}
\end{gathered}
$$

Por último a distância da face à origem é definida pela Equação 13, em que $x_{c}, y_{c}$ e $z_{c}$ são as coordenadas do baricentro de cada face. Tais coordenadas são calculadas pelas Equações 14,15 e 16 , nas quais $x_{n}, y_{n}$ e $z_{n}$ indicam as coordenadas cartesianas do vértice $n$ da face.

$$
\begin{aligned}
D_{k} & =\sqrt{x_{c}^{2}+y_{c}^{2}+z_{c}^{2}} \\
x_{c} & =\frac{x_{1}+x_{2}+x_{3}}{3} \\
y_{c} & =\frac{y_{1}+y_{2}+y_{3}}{3} \\
z_{c} & =\frac{z_{1}+z_{2}+z_{3}}{3}
\end{aligned}
$$




\subsection{Método para comparação entre dois modelos}

O procedimento de comparação entre dois modelos é ilustrado na Figura 3. Após calcular os GGFMs de dois modelos, os valores de área e distância de cada face são mapeados em matrizes com índices equivalentes às coordenadas esféricas do vetor normal da face [17].

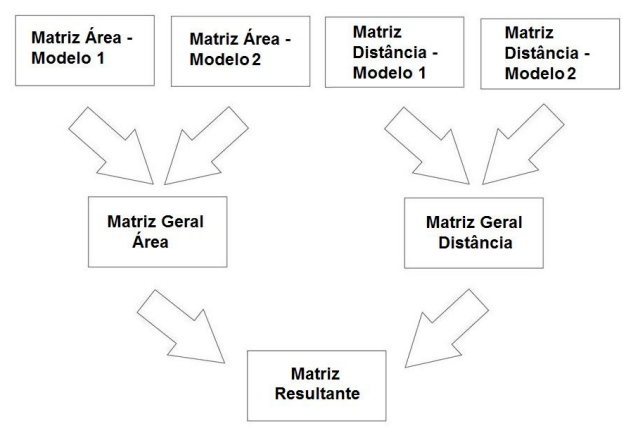

Figura 3. Esquema de como os modelos são comparados

Ao se comparar dois GGFMs, são obtidas quatro matrizes que armazenam: áreas das faces do primeiro modelo, áreas das faces do segundo modelo, distâncias das faces do primeiro modelo e distâncias das faces do segundo modelo. As linhas e colunas das matrizes são indexadas, respectivamente, por $\theta$ e $\phi$ arredondados em intervalos de $90^{\circ}$. Cada célula da matriz armazena o somatório da característica extraída - área ou distância - das faces do modelo, no intervalo citado.

Após mapeadas as áreas e distâncias nas quatro primeiras matrizes, a Equação 17 é utilizada para definir a matriz geral de áreas dos dois modelos, na qual $M G A_{i j}$ corresponde ao valor da célula $i j$ dessa matriz, $M A 1_{i j}$ corresponde ao valor da célula $i j$ da matriz de áreas do primeiro modelo e $M A 2_{i j}$ corresponde ao valor da célula $i j$ da matriz de áreas do segundo modelo.

$$
M G A_{i j}=\left(M A 1_{i j}-M A 2_{i j}\right)^{2}
$$

De forma similar, a Equação 18 define a matriz geral de distâncias dos dois modelos, na qual $M G D_{i j}$ corresponde ao valor da célula $i j$ dessa matriz, $M D 1_{i j}$ corresponde ao valor da célula $i j$ da matriz de distâncias do primeiro modelo e $M D 2_{i j}$ corresponde ao valor da célula $i j$ da matriz de distâncias do segundo modelo. 


$$
M G D_{i j}=\left(M D 1_{i j}-M D 2_{i j}\right)^{2}
$$

Em seguida, define-se a matriz resultante do processo, segundo a Equação 19, na qual MatRes $_{i j}$ corresponde ao valor da célula $i j$ dessa matriz e os demais termos foram definidos anteriormente.

$$
\text { MatRes }_{i j}=\sqrt{M G A_{i j}+M G D_{i j}}
$$

A distância total (DT) entre os dois modelos é obtida pela soma das células da matriz final, conforme a Equação 20, em que $i$ e $j$ correspondem aos valores de linha e coluna da célula e $n$ e $m$ correspondem aos valores máximos que essas variáveis podem assumir.

$$
D T=\sum_{i=1}^{n} \sum_{j=1}^{m} \text { MatRes }_{i j}
$$

\subsection{Experimentos}

A avaliação do ELAD foi conduzida por dois experimentos: (a) uso de modelos genéricos, com a finalidade de comprovar a adequação da concepção e da implementação do extrator e (b) modelos cardíacos do ventrículo esquerdo reconstruídos a partir de imagens de Ressonância Magnética Nuclear.

4.3.1 Modelos Genéricos Na Figura 4 são apresentados exemplos dos modelos genéricos construídos. Para conferir a adequação do extrator, os testes buscavam identificar intensidades diferentes de uma deformação previamente conhecida, inserida propositalmente nos modelos 3D. Foram usados cinco modelos genéricos com vértices e faces previamente conhecidos. Na Tabela 1 são apresentados os valores das coordenadas de cada vértice dos modelos da Figura 4, assim como os vértices que compõem cada face. A coluna "faces" indica os vértices que foram a respectiva face. Os modelos possuem centróide localizado na origem do plano e as deformações 1 e 2 foram aplicadas no vértice 4, com variação da intensidade da deformação. Os demais vértices permanecem iguais ao modelo original. Dessa forma, é possível conhecer exatamente a localização e a intensidade da deformação e, assim, validar o extrator desenvolvido.

4.3.2 Modelos Cardíacos Foram utilizados 30 modelos cardíacos do ventrículo esquerdo reconstruídos a partir de exames de Ressonância Magnética Nuclear provenientes do Instituto 


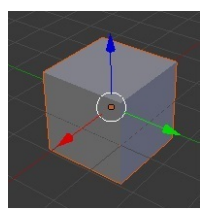

(a)

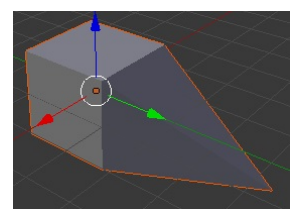

(b)

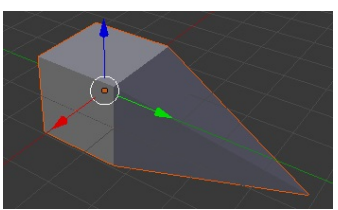

(c)

Figura 4. Modelos genéricos empregados nos experimentos: (a) modelo Normal; (b) modelo Deformado1; (c) modelo Deformado2.

\begin{tabular}{|c|c|c|c|c|c|c|c|c|c|c|c|c|}
\hline & \multicolumn{4}{|c|}{ Normal } & \multicolumn{4}{|c|}{ Deformado 1} & \multicolumn{4}{|c|}{ Deformado 2} \\
\hline & \multicolumn{3}{|c|}{ Vértices } & \multirow[t]{2}{*}{ Faces } & \multicolumn{3}{|c|}{ Vértices } & \multirow[t]{2}{*}{ Faces } & \multicolumn{3}{|c|}{ Vértices } & \multirow[t]{2}{*}{ Faces } \\
\hline & $\mathbf{X}$ & $\mathbf{Y}$ & $\mathbf{Z}$ & & $\mathbf{X}$ & $\mathbf{Y}$ & $\mathbf{Z}$ & & $\mathbf{X}$ & $\mathbf{Y}$ & $\mathbf{Z}$ & \\
\hline 1 & 1 & -1 & -1 & 123 & 1 & -1 & -1 & 123 & 1 & -1 & -1 & 123 \\
\hline 2 & 1 & -1 & 1 & 124 & 1 & -1 & 1 & 124 & 1 & -1 & 1 & 124 \\
\hline 3 & -1 & -1 & 1 & 587 & -1 & -1 & 1 & 587 & -1 & -1 & 1 & 587 \\
\hline 4 & -1 & -1 & -1 & 576 & -3 & -3 & -3 & 576 & -4 & -4 & -4 & 576 \\
\hline 5 & 1 & 1 & -1 & 156 & 1 & 1 & -1 & 156 & 1 & 1 & -1 & 156 \\
\hline 6 & 1 & 1 & 1 & 162 & 1 & 1 & 1 & 162 & 1 & 1 & 1 & 162 \\
\hline 7 & -1 & 1 & 1 & 267 & -1 & 1 & 1 & 267 & -1 & 1 & 1 & 267 \\
\hline 8 & -1 & 1 & -1 & 273 & -1 & 1 & -1 & 273 & -1 & 1 & -1 & 273 \\
\hline 9 & & & & 378 & & & & 378 & & & & 378 \\
\hline 10 & & & & 384 & & & & 384 & & & & 384 \\
\hline 11 & & & & 514 & & & & 514 & & & & 514 \\
\hline 12 & & & & 548 & & & & 548 & & & & 548 \\
\hline
\end{tabular}

Tabela 1. Coordenadas dos vértices e faces de cada modelo da Figura 4.

do Coração (InCor). As Figuras 5 (a) e 5 (b) apresentam um exemplo do processo de segmentação realizada nas imagens médicas, cada exame possuia 45 slices que foram segmentados manualmente utilizando o software Seg3D [9].

As Figuras 6 e 7 apresentam exemplos dos modelos cardíacos dos ventrículos esquerdos utilizados e já com a superfície 3D reconstruída pelo software ImageVis [8]. Estes casos foram pré-classificados por especialistas médicos como contendo ou não a doença de ICC. A Figura 6 apresenta exemplos de casos em que os pacientes são saudáveis. Na Figura 7 são disponibilizados casos de pacientes que possuem a doença de ICC. É possível notar que as diferenças entre os modelos com e sem ICC são bem sutis e a olho nu não é possível diferenciar cada caso. No dia a dia o mais comum é que o especialista médico analise diversos exames 
como Eletrocardiografia, Ressonância Magnética Nuclear e o próprio histórico do paciente para formar o diagnóstico de ICC.

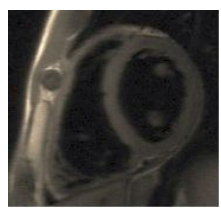

(a)

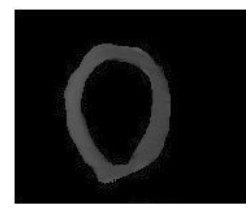

(b)

Figura 5. Exemplo de slice do ventrículo esquerdo que foi segmentado: (a) imagem original proveniente do exame de Ressonância Magnética Nuclear; (b) Parede ventricular segmentada .
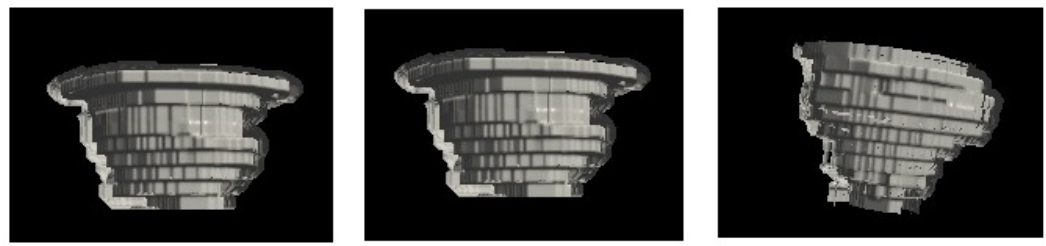

Figura 6. Exemplo de modelos cardíacos sem a presença de ICC.
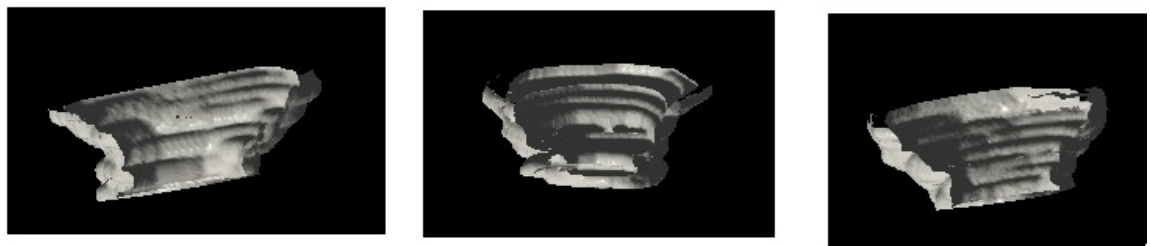

Figura 7. Exemplo de modelos cardíacos com ICC.

\subsection{Avaliação}

Para a avaliação do ELAD, foram utilizadas a Matriz de Confusão e a métrica de Precisão vs. Revocação. A Matriz de Confusão ilustra por meio de uma escala de cores o quão semelhante o modelo é daquele fornecido como consulta. As células foram coloridas de forma que as de menores distâncias têm cor mais escura. Foram definidos cinco intervalos de distância (DT) - e, consequentemente, cinco níveis de cinza diferentes, a saber: (1) DT =0; 
(2) $0<$ DT $\leq 1,5$; (3) $1,5<$ DT $\leq 2,25$; (4) $2,25<$ DT $\leq 3$ e (5) distância $>3$, 0 . Ao primeiro grupo foi atribuída a cor preta e ao último grupo foi atribuída a cor branca. Aos demais foram atribuídos níveis de cinza intermediários.

O gráfico de Precisão vs. Revocação é uma forma muito utilizada para avaliar sistemas de CBIR. A precisão (Equação 21) indica a proporção de imagens relevantes que foram recuperadas, enquanto a revocação (Equação 22) é a proporção entre as imagens relevantes presentes no banco de dados e que foram recuperadas. Uma forma de comparar o desempenho entre sistemas diferentes é calcular a área sob a curva obtida.

$\frac{\text { ImagensRelevantes } \cap \text { ImagensRecuperadas }}{\text { ImagensRecuperadas }}$
$\frac{\text { ImagensRelevantes } \cap \text { ImagensRecuperadas }}{\text { ImagensRelevantes }}$

\section{Resultados e discussão}

\subsection{Modelos genéricos}

O ELAD foi aplicado no modelos apresentados na Seção 4.3.1. Para exemplificar o resultado de cada passo da técnica, comprovando sua adequação, as matrizes de áreas e distâncias resultantes das comparações entre os modelos apresentados na Figura 4 são disponibilizadas a seguir. A Tabela 2 apresenta os resultados da comparação entre o modelo Normal e o modelo Deformado1. Na Tabela 3 estão disponibilizados os resultados da comparação entre o modelo Normal e o modelo Deformado2 e na Tabela 4 são apresentadas as matrizes resultantes da comparação entre os dois modelos deformados.

Nas tabelas citadas, as células com valor zero indicam que os dois modelos possuem valor igual de área ou distância naquela região, já as células com valores diferentes de zero mostram que existe uma diferença naquela região entre os dois modelos comparados. Nota-se que os valores dessas células são maiores na Tabela 3 do que na Tabela 2, devido à deformação mais acentuada no modelo Deformado2. Na Tabela 4 observam-se valores menores do que nas demais tabelas, indicando que a diferença entre os dois modelos deformados é menor que a diferença entre cada modelo deformado e o modelo normal.

As matrizes finais resultantes de cada comparação são apresentadas nas Tabelas 5, 6 e 7. Conforme descrito na Seção 4, as células de cada matriz são somadas para obter a distância efetiva entre os dois modelos comparados. Os valores obtidos nessas tabelas mostram a capacidade do extrator detectar e comparar as características do modelo de forma local, indicando diferenças nas regiões afetadas pelo aumento da intensidade da deformação (células $(90,-90),(90,180),(180,0)$ e $(180,90)$ e indicando que os modelos são iguais nas 
Recuperação de modelos médicos por conteúdo usando Extrator Local de Área e Distância

\begin{tabular}{|c||c|c|c|c|c|}
\hline \multicolumn{5}{|c|}{ Matriz de áreas - Normal X Deformado1 } \\
\hline theta/phi & $\mathbf{- 1 8 0}$ & $\mathbf{- 9 0}$ & $\mathbf{0}$ & $\mathbf{9 0}$ & $\mathbf{1 8 0}$ \\
\hline $\mathbf{0}$ & & & 0 & & \\
\hline $\mathbf{9 0}$ & & 21,5 & 0 & 0 & 21,5 \\
\hline $\mathbf{1 8 0}$ & & & 0,2 & 20 & \\
\hline
\end{tabular}

\begin{tabular}{|c||c|c|c|c|c|}
\hline \multicolumn{5}{|c|}{ Matriz de distâncias - Normal X Deformado1 } \\
\hline theta/phi & $\mathbf{- 1 8 0}$ & $\mathbf{- 9 0}$ & $\mathbf{0}$ & $\mathbf{9 0}$ & $\mathbf{1 8 0}$ \\
\hline $\mathbf{0}$ & & & 0 & & \\
\hline $\mathbf{9 0}$ & & 1,2 & 0 & 0 & 1,2 \\
\hline $\mathbf{1 8 0}$ & & & 0,1 & 3,9 & \\
\hline
\end{tabular}

Tabela 2. Matrizes gerais de área e distância da comparação entre os modelos Normal e Deformado1.

\begin{tabular}{|c||c|c|c|c|c|}
\hline \multicolumn{5}{|c|}{ Matriz de áreas - Normal X Deformado2 } \\
\hline theta/phi & $\mathbf{- 1 8 0}$ & $\mathbf{- 9 0}$ & $\mathbf{0}$ & $\mathbf{9 0}$ & $\mathbf{1 8 0}$ \\
\hline $\mathbf{0}$ & & & 0 & & \\
\hline $\mathbf{9 0}$ & & 49,8 & 0 & 0 & 49,8 \\
\hline $\mathbf{1 8 0}$ & & & 3,4 & 34 & \\
\hline
\end{tabular}

\begin{tabular}{|c||c|c|c|c|c|}
\hline \multicolumn{6}{|c|}{ Matriz de distâncias - Normal X Deformado2 } \\
\hline theta/phi & $\mathbf{- 1 8 0}$ & $\mathbf{- 9 0}$ & $\mathbf{0}$ & $\mathbf{9 0}$ & $\mathbf{1 8 0}$ \\
\hline $\mathbf{0}$ & & & 0 & & \\
\hline $\mathbf{9 0}$ & & 2,7 & 0 & 0 & 2,7 \\
\hline $\mathbf{1 8 0}$ & & & 0,1 & 6,2 & \\
\hline
\end{tabular}

Tabela 3. Matrizes gerais de área e distância da comparação entre os modelos Normal e Deformado2.

\begin{tabular}{|c||c|c|c|c|c|}
\hline \multicolumn{5}{|c|}{ Matriz área - Deformado1 X Deformado2 } \\
\hline theta/phi & $\mathbf{- 1 8 0}$ & $\mathbf{- 9 0}$ & $\mathbf{0}$ & $\mathbf{9 0}$ & $\mathbf{1 8 0}$ \\
\hline $\mathbf{0}$ & & & 0 & & \\
\hline $\mathbf{9 0}$ & & 5,9 & 0 & 0 & 5,9 \\
\hline $\mathbf{1 8 0}$ & & & 1,8 & 1,8 & \\
\hline
\end{tabular}

\begin{tabular}{|c||c|c|c|c|c|}
\hline \multicolumn{6}{|c|}{ Matriz distância - Deformado1 X Deformado2 } \\
\hline theta/phi & $\mathbf{- 1 8 0}$ & $\mathbf{- 9 0}$ & $\mathbf{0}$ & $\mathbf{9 0}$ & $\mathbf{1 8 0}$ \\
\hline $\mathbf{0}$ & & & 0 & & \\
\hline $\mathbf{9 0}$ & & 0,3 & 0 & 0 & 0,3 \\
\hline $\mathbf{1 8 0}$ & & & 0,3 & 0,3 & \\
\hline
\end{tabular}

Tabela 4. Matrizes gerais de área e distância da comparação entre os modelos Deformado1 e Deformado2.

regiões não afetadas (células $(0,0),(90,0)$ e $(90,90))$. A última linha (Soma) apresenta o valor total das distâncias entradas.

\begin{tabular}{|c||c|c|c|c|c|}
\hline theta/phi & $\mathbf{- 1 8 0}$ & $\mathbf{- 9 0}$ & $\mathbf{0}$ & $\mathbf{9 0}$ & $\mathbf{1 8 0}$ \\
\hline \hline $\mathbf{0}$ & & & 0 & & \\
\hline $\mathbf{9 0}$ & & 4,8 & 0 & 0 & 4,8 \\
\hline $\mathbf{1 8 0}$ & & & 0,5 & 4,9 & \\
\hline Soma & 15 & & & & \\
\hline
\end{tabular}

Tabela 5. Matriz obtida da comparação entre os modelos Normal e Deformado1. 
Recuperação de modelos médicos por conteúdo usando Extrator Local de Área e Distância

\begin{tabular}{|c||c|c|c|c|c|}
\hline theta/phi & $\mathbf{- 1 8 0}$ & $\mathbf{- 9 0}$ & $\mathbf{0}$ & $\mathbf{9 0}$ & $\mathbf{1 8 0}$ \\
\hline \hline $\mathbf{0}$ & & & 0 & & \\
\hline $\mathbf{9 0}$ & & 7,2 & 0 & 0 & 7,2 \\
\hline $\mathbf{1 8 0}$ & & & 1,9 & 6,3 & \\
\hline Soma & 22,6 & & & & \\
\hline
\end{tabular}

Tabela 6. Matriz obtida da comparação entre os modelos Normal e Deformado2.

\begin{tabular}{|c||c|c|c|c|c|}
\hline theta/phi & $\mathbf{- 1 8 0}$ & $\mathbf{- 9 0}$ & $\mathbf{0}$ & $\mathbf{9 0}$ & $\mathbf{1 8 0}$ \\
\hline \hline $\mathbf{0}$ & & & 0 & & \\
\hline $\mathbf{9 0}$ & & 2,5 & 0 & 0 & 2,5 \\
\hline $\mathbf{1 8 0}$ & & & 1,5 & 1,5 & \\
\hline Soma & 8 & & & & \\
\hline
\end{tabular}

Tabela 7. Matriz obtida da comparação entre os modelos Deformado1 e Deformado2.

As distâncias finais obtidas foram usadas para obter a matriz de confusão (Tabela 8), que mostra as comparações entre os diferentes modelos. Os modelos Deformado3 e Deformado4, que não foram detalhados anteriormente, possuem a mesma deformação local dos modelos Deformado1 e Deformado2, porém com maior intensidade. Esta tabela comprova a adequação da concepção e da implementação do ELAD, pois foram obtidos resultados coerentes com a teoria envolvida. Uma vez que conhecia-se previamente todos os vértices e faces dos modelos 3D construídos, foi possível verificar que o extrator retornou distâncias que refletem as diferenças existentes entre os modelos 3D. Verifica-se que a distância do modelo 3D normal para os modelos 3D deformados $(1,2,3$ e 4$)$ é crescente e constante, proporcional à intensidade das deformações neles inseridas.

Conforme mencionado, nos modelos sintéticos discutidos nesta seção as deformações foram artificialmente e controladamente inseridas com a finalidade de validar a abordagem proposta. Verifica-se que ao medir o total das áreas das faces dentro de um determinado intervalo (no caso, de $90^{\circ} \mathrm{em} 90^{\circ}$ ) a abordagem permite verificar se um modelo possui área maior que outro naquele intervalo. No entanto, é possível que o mesmo modelo tenha coincidentemente a mesma área no mesmo intervalo, mas resultante de uma topologia diferenciada das faces. Isso resultaria na indicação de dois modelos diferentes como sendo iguais. Na abordagem proposta, este problema é solucionado acrescentando a distância das faces ao centróide como a segunda característica a ser analisada. A soma dessas distâncias permite diferenciar topologias desiguais e, portanto, diferenciar deformações locais. Verifica-se que ao utilizar a soma total das duas características (área e distância) até mesmo diferenças sutis na forma dos 
modelos podem ser detectadas, como será visto com os modelos cardíacos reais, na próxima seção.

\begin{tabular}{|c||c|c|c|c|c|}
\hline & Normal & Deformado 1 & Deformado 2 & Deformado 3 & Deformado 4 \\
\hline \hline Normal & 0,0 & 14,9 & 22,7 & 30,6 & 38,6 \\
\hline Deformado 1 & 14,9 & 0,0 & 7,9 & 15,8 & 23,8 \\
\hline Deformado 2 & 22,7 & 7,9 & 0,0 & 7,9 & 15,9 \\
\hline Deformado 3 & 30,6 & 15,8 & 7,9 & 0,0 & 8,0 \\
\hline Deformado 4 & 38,6 & 23,8 & 15,9 & 8,0 & 0,0 \\
\hline
\end{tabular}

Tabela 8. Matriz de confusão resultante da comparação entre os modelos.

\subsection{Modelos cardíacos tridimensionais}

Como mencionado na Seção 4.3.2, os modelos cardíacos foram divididos em dois grupos: modelos com ICC e modelos sem ICC. A fim de verificar a precisão dos resultados foi construída a Matriz de Confusão apresentada na Figura 8, na qual são destacados quatro clusters, localizados nos quatro quadrantes. O primeiro quadrante (superior esquerdo) indica as comparações entre dois modelos diagnosticados com ICC, o segundo e o terceiro quadrantes (superior direito e inferior esquerdo) indicam comparações entre um modelo com ICC e outro sem a doença e o quarto quadrante indica comparações entre dois modelos sem ICC. Dessa forma, espera-se que o primeiro e o quarto quadrantes estejam com células mais escuras do que os demais, indicando que as distâncias entre casos similares são menores. De fato, percebe-se que isso ocorre na Figura 8, indicando que o ELAD fornece uma boa precisão na recuperação de modelos similares.

Pode-se atribuir esse desempenho ao fato do extrator ter a capacidade de analisar a área e a distância ao centróide das faces do modelo de forma local, justamente porque considera a orientação do vetor normal da face. Dessa forma, as regiões deformadas são detectadas e comparadas diretamente com a mesma região de outros modelos. $\mathrm{O}$ extrator consegue dividir o modelo em várias regiões de comparação, aumentando a possibilidade de diferenciação, de forma que modelos considerados similares possuem mais regiões similares entre si, o que faz com que aquele modelo seja recuperado corretamente.

Em particular, é interessante destacar as situações do Paciente 26 e do Paciente 21 como as mais incorretas. Esses casos foram recuperados como sendo semelhantes aos modelos diagnosticados com ICC, quando, na verdade, eles não possuem a doença. Apesar disso, esses casos não foram recuperados com a menor distância em relação aos caso diagnosticados com ICC. 


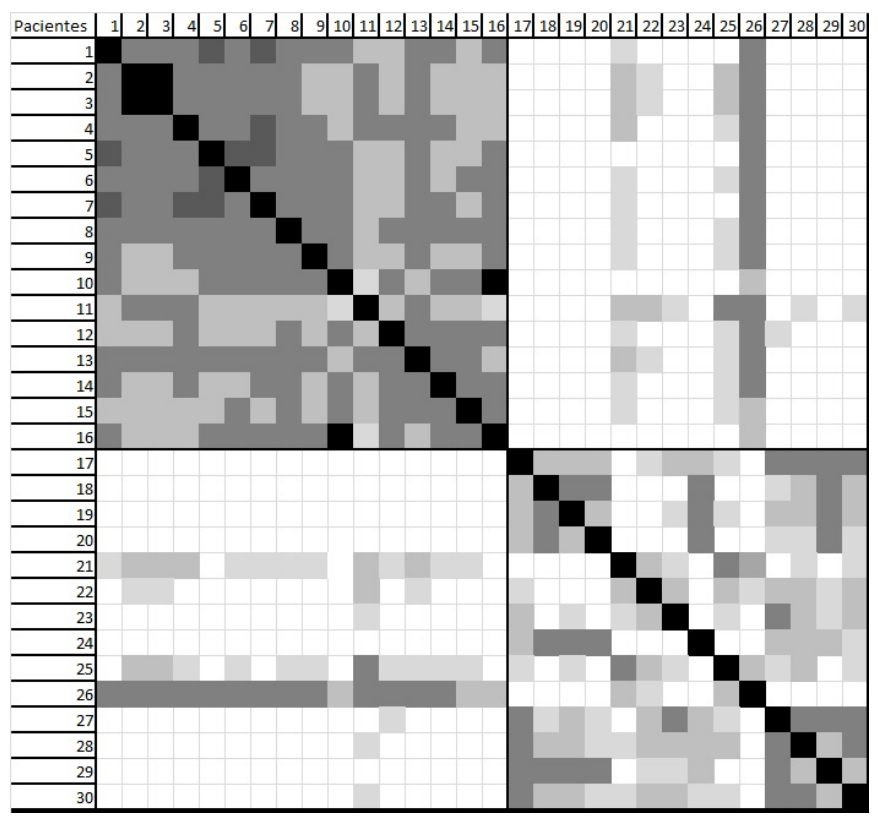

Figura 8. Matriz de confusão resultante da comparação entre os modelos cardíacos

É notável, também, a maior sensibilidade do extrator em identificar semelhança entre os modelos com ICC do que semelhança entre os modelos considerados normais, característica evidenciada pelo fato do primeiro cluster ser mais "escuro"do que o quarto cluster. Isso deve-se ao fato de que a ICC caracteriza-se pela deformação localizada do ventrículo esquerdo, motivo pelo qual o emprego de um extrator de forma com características locais, como o ELAD, mostra-se adequado.

Finalmente deve ser observado que o ELAD apresentou menor eficiência quando o tamanho dos intervalos das matrizes de comparação dos modelos foi diminuído. $\mathrm{O}$ ato de diminuir o intervalo pode ser necessário em casos que a deformação é muito sutil e em regiões diminutas do modelo. Assim, o ELAD ainda precisa de melhorias para atender a esse tipo de caso.

A Figura 9 mostra os gráficos de Precisão vs. Revocação para os modelos cardíacos, com e sem ICC. Nos gráficos apresentados é possível comparar o desempenho do ELAD com o desempenho de um extrator baseado na Transformada de Hough 3D (ETH3D), desenvolvido em um trabalho anterior [2]. Os gráficos indicam que a precisão do ELAD se manteve alta mesmo com valores altos de revocação, ao passo que o ETH3D apresentou uma queda 
de desempenho considerável ao se aumentar os valores de revocação. Visualmente é possível perceber que a área entre a curva do ELAD com o eixo $x$ é maior que a área referente ao ETH3D. Tanto para casos com ICC quanto para casos sem a doença, o desempenho do ELAD mostrou-se superior ao ETH3D, ratificando os resultados apresentados na seção anterior, que fornecem indícios da precisão do ELAD como uma ferramenta de auxílio ao diagnóstico de ICC.
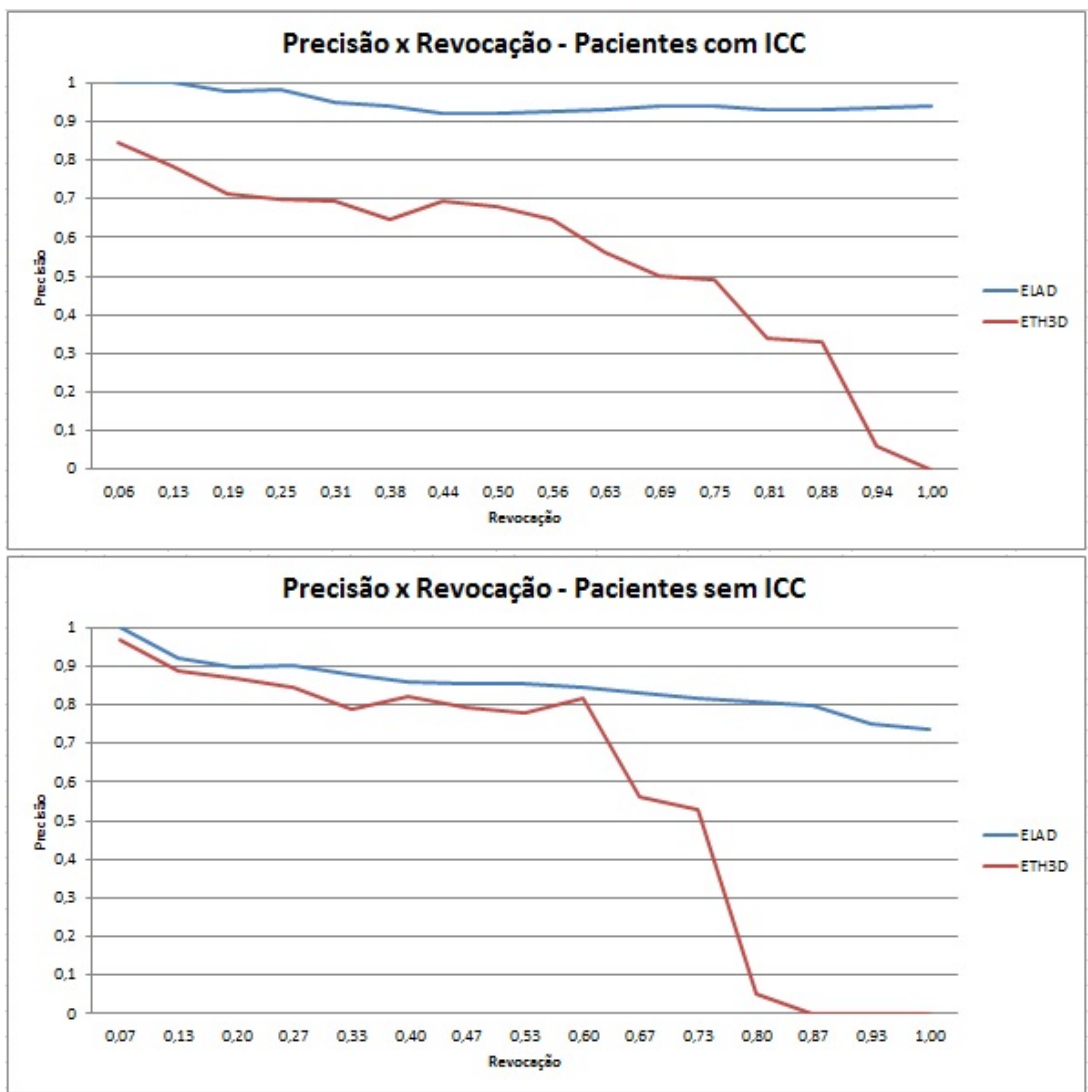

Figura 9. Gráfico de Precisão vs. Revocação da comparação do desempenho do ELAD e ETH3D. 


\section{Conclusões}

Nesse artigo foi apresentado o extrator ELAD, juntamente com um novo método de comparar modelos tridimensionais visando a recuperação por conteúdo de modelos médicos 3D. Seu desempenho foi comparado com o do extrator ETH3D para recuperar modelos cardíacos do ventrículo esquerdo, como forma de auxílio ao diagnóstico de ICC. Foi obtida uma média de precisão de $93 \%$ para modelos diagnosticados com ICC para todos os valores de Revocação e nos casos sem ICC a média de precisão ficou em 75\%, indicando um desempenho superior observado no extrator ETH3D que obteve precisão média de $75 \%$ para casos com ICC e $65 \%$ para casos sem ICC.

O ELAD mostrou-se eficiente para detectar deformações nos modelos por considerar características como área e posicionamento de cada face, que fornecem uma visão completa do modelo 3D. A implementação aqui realizada imprimiu ao extrator apresentado a capacidade de detectar deformações localizadas. Esta capacidade é falha em extratores globais, mas é essencial quando deseja-se construir aplicações cujas formas dos modelos são alteradas apenas localmente, como o estudo de caso aqui apresentado - diagnóstico de ICC. Assim, os resultados iniciais indicam que o ELAD pode ser uma abordagem eficiente para compor Sistemas de Auxílio ao Diagnóstico com essas particularidades.

Como trabalhos futuros, pretende-se realizar novos testes atribuindo pesos diferentes às métricas de área e distância das faces para identificar se existe uma melhora no desempenho do extrator, visto que as medidas de área são mais sensíveis à deformação do modelo. Planeja-se aperfeiçoar o ELAD para aumentar sua eficiência em modelos com deformações em pequenas regiões, assim como avaliar o extrator para outros modelos não convexos.

\section{Agradecimentos}

Ao Programa de Educação Tutorial do Ministério da Educação; à Prof ${ }^{\mathrm{a}} \operatorname{Dr}^{\mathrm{a}}$ Sarajane Marques Peres, tutora do grupo PET-SI; ao Dr. Carlos Rochitte (Incor-SP) pelo fornecimento das imagens médicas, ao Conselho Nacional de Desenvolvimento Científico e Tecnológico (CNPq) - Processo 401745/2013-9 e ao Instituto Nacional de Ciência e Tecnologia - Medicina Assistida por Computação Científica (INCT-MACC), pelo apoio financeiro.

\section{Referências}

[1] Aman, J., Yao, J. e Summers, R. Content-based image retrieval on CT colonography using rotation and scale invariant features and bag-of-words model. In Proceedings of 2th IEEE International Symposium on Biomedical Imaging: From Nano to Macro (Antalya, Turkey., april 2010), IEEE Computer Society, pp. 1357 -1360. 
[2] Bergamasco, L. C. Recuperação de imagens cardíacas por conteúdo. Dissertação de Mestrado, Universidade de São Paulo, 2013.

[3] Bergamasco, L. C. E Nunes, F. L. Applying distance histogram to retrieve 3d cardiac medical models. In AMIA Annual Symposium Proceedings (2013), vol. 2013, American Medical Informatics Association, p. 112.

[4] Bergamasco, L. C. E Nunes, F. L. A new local feature extraction approach for content-based $3 \mathrm{~d}$ medical model retrieval using shape descriptor. In Proceedings of the 29th Annual ACM Symposium on Applied Computing (2014), ACM, pp. 902-907.

[5] Böhm, C., Berchtold, S. E Keim, D. A. Searching in high-dimensional spaces: Index structures for improving the performance of multimedia databases. ACM Computing Surveys (CSUR) 33, 3 (2001), 322-373.

[6] Bugatti, P. H. Análise da influência de funçoes de distância para o processamento de consultas por similaridade em recuperaçao de imagens por conteúdo. Tese de Doutorado, Universidade de São Paulo, 2008.

[7] Castanón, C. A. B. Recuperaçao de imagens por conteúdo através de análise multiresoluçao por wavelets. Tese de Doutorado, Universidade de São Paulo, 2003.

[8] CBIC. ImageVis3D: A Real-time Volume Rendering Tool for Large Data. Scientific Computing and Imaging Institute (SCI), 2012. Disponível em:<http://www.imagevis3D.org $>$.

[9] CBIC. Seg3D: Volumetric Image Segmentation and Visualization. Scientific Computing and Imaging Institute (SCI), 2012. Disponível em: <http://www.seg3D.org>.

[10] Chen, Z. Q., Zou, K. S., IP, W. H. E Chan, C. Y. 3d model retrieval based on fuzzy weighted shape distributions. Advanced Materials Research 201 (2011), 1678-1681.

[11] Da Silva Torres, R. E FAlCão, A. X. Content-based image retrieval: Theory and applications. RITA 13, 2 (2006), 161-185.

[12] Depeursinge, A., Foncubierta-Rodriguez, A., Van De Ville, D. E MülLER, H. Three-dimensional solid texture analysis in biomedical imaging: Review and opportunities. Medical image analysis 18, 1 (2014), 176-196.

[13] DoI, K. Computer-aided diagnosis in medical imaging: historical review, current status and future potential. Computerized medical imaging and graphics 31, 4-5 (2007), 198211. 
Recuperação de modelos médicos por conteúdo usando Extrator Local de Área e Distância

[14] Glatard, T., Montagnat, J. E Magnin, I. E. Texture based medical image indexing and retrieval: application to cardiac imaging. In Proceedings of the 6th ACM SIGMM international workshop on Multimedia information retrieval (New York, NY, USA, 2004), MIR '04, ACM, pp. 135-142.

[15] Gong, B., XU, C., LiU, J. E TANG, X. Boosting 3d object retrieval by object flexibility. In Proceedings of the 17th ACM international conference on Multimedia (2009), ACM, pp. 525-528.

[16] Horn, B. K. P. Extended gaussian images. Proceedings of the IEEE 72, 12 (1984), 1671-1686.

[17] KASPRZAK, P. E KowAlCZUK, P. Objects recognition with high-resolution insar data and global geometric feature map. In Signal Processing Symposium (SPS), 2013 (2013), IEEE, pp. 1-5.

[18] KhoshelHAM, K. Extending generalized hough transform to detect 3d objects in laser range data, 2007.

[19] Kumar, A., Kim, J., Wen, L. E FenG, D. A graph-based approach to the retrieval of volumetric pet-ct lung images. In Engineering in Medicine and Biology Society (EMBC), 2012 Annual International Conference of the IEEE (2012), IEEE, pp. 54085411 .

[20] LU, K., ZhaO, F. E He, N. An effective approach to content-based 3d model retrieval and classification. In Computational Intelligence and Security, 2007 International Conference on (2007), IEEE, pp. 361-365.

[21] Marques Filho, O. E Vieira Neto, H. Processamento digital de imagens. Brasport, 1999.

[22] Murala, S. E Wu, Q. J. Mri and ct image indexing and retrieval using local mesh peak valley edge patterns. Signal Processing: Image Communication 29, 3 (2014), 400-409.

[23] Nunes, F. D. L. D. S. Processamento gráfico para aplicações em saúde: técnicas, requisitos, ferramentas, desafios e oportunidades. Tese de Doutorado, Universidade de São Paulo, 2011.

[24] Smeulders, A. W., Worring, M., Santini, S., Gupta, A. E Jain, R. Contentbased image retrieval at the end of the early years. Pattern Analysis and Machine Intelligence, IEEE Transactions on 22, 12 (2000), 1349-1380. 
[25] WAng, D., CUI, C. E WU, Z. Matching 3d models with global geometric feature map. In Multi-Media Modelling Conference Proceedings, 2006 12th International (2006), IEEE, pp. 4-pp.

[26] Wu, H., Kim, J., CAI, W. E FEnG, D. Volume of interest (VOI) feature representation and retrieval of multi-dimensional dynamic positron emission tomography images. In Intelligent Multimedia, Video and Speech Processing, 2004. Proceedings of 2004 International Symposium on (Hong Kong, China, oct. 2004), IE, pp. 639 - 642. 